\title{
INTRODUCCIÓN A LA EMPRESA FAMILIAR
}

Leonardo Centeno Caffarena

Ingeniero industrial

Coordinador Proyecto UNI/BID

leonardo.centeno@uni.edu.ni

\section{RESUMEN}

La empresa familiar es el tipo de empresa más numeroso que hay en cualquier país, sea éste uno desarrollado o en vías de desarrollo. Su objetivo inicial es servir de fuente de empleo para la familia empresaria, y de esta manera satisfacer sus necesidades laborales y económicas. Sin embargo, la empresa familiar cuenta con el reto de sobrevivir a la 3ra o 4ta generación, que es cuando desaparecen entre el 85 y el 95\% de las unidades económicas de este tipo. En este trabajo expondremos las características y retos de estas empresas, enfatizando en los tipos de empresas familiares que existen. Al final expondremos un modelo innovador en la región latinoamericana, que la UNI está empezando a implementar en Nicaragua, para asegurarnos que los negocios familiares puedan sobrepasar la barrera que limita su sobrevivencia con relación al tiempo y poder llevarla con éxito bajo los retos que le establece el siglo XXI.

Palabras claves: Empresa familiar, firma familiar, negocio familiar, finca familiar

\section{INTRODUCCIÓN}

La empresa familiar es probablemente tan antigua como la familia misma. Siempre que ésta última desarrolla alguna capacidad económica, vía algún tipo de negocio, tarde o temprano se verá en la necesidad de involucrar a la sub-siguiente generación, primero para que tenga una ocupación, pero la medida en que el negocio se hace más rentable, y que las capacidades del fundador van disminuyendo hasta su desaparición, finalmente sus sucesores terminan tomando el control total del negocio.

Para efectos de este artículo, cuando nos referimos a la empresa familiar, nos estaremos refiriendo indistintamente a la finca familiar, un tipo de negocio familiar que se ha estudiado menos, pero que tiene sus características propias, por ejemplo es una unidad económica estable, físicamente, pues permanece en el campo, diferente a la empresa urbana, que tiene mayor facilidad de movimiento.

\section{La problemática}

Los estudios empíricos nos muestran que las empresas familiares (EFs) tienen problemas de sobrevivencia, especialmente entre las 3ra y 4 ta generación; datos internacionales indican que $35 \%$ de las EFs de cualquier país sufren, en todo momento, un proceso de sucesión, y que sólo un $20 \%$ de ellas tiene un plan de sucesión; el $40 \%$ alcanza la 2da generación, el 15\% la 3ra generación, y sólo el 4.5\% llega a la 4ta generación (Ward, 1997).

El problema anterior tiene varias causas, entre las que se pueden mencionar: el estilo de administración del negocio, los temas relacionados a la sucesión, el rol y el estilo del fundador, confusión entre los roles de propietario y gerente, confusión entre el interés de la familia y de la empresa, atraso en el proceso de sucesión, crecimiento e internacionalización de la empresa, desarrollo de sucesores, conflictos entre miembros familiares, la planificación estratégica del negocio, temas de riqueza y propiedad, la profesionalización, gobierno corporativo, y enfoque de género, entre otros. 


\section{IMPORTANCIA DE LA EMPRESA FAMILIAR}

La empresa familiar es el tipo de unidad económica más abundante en la tierra, y así siempre ha sido, pues tarde o temprano los empresarios se van involucrando y terminan sucediendo el negocio a sus familiares.

En los Estados Unidos, el país que más se ha estudiado el fenómeno de la empresa familiar se sabe que el $20 \%$ de las 500 más grandes son familiares con $90 \%$ de ellas con parientes en posiciones gerenciales (Donnelley, 1963), del 95-97\% de las firmas registradas son familiares, representan del $40-50 \%$ del PIB del país y generan entre el $40-$ $42 \%$ del empleo.

En España, el segundo país que más se ha estudiado, se sabe que las empresas familiares representan el $71 \%$ de las empresas con ventas mayores a los US $\$ 2$ millones, $61 \%$ de las ventas, $62 \%$ del empleo y 59 de las exportaciones del país (Gallo $\&$ Garcia Pont, 1989).

Otros estudios han revelado que el $50 \%$ de la fuerza laboral de Australia es generada por las empresas familiares y en Europa del Este entre 45 y $65 \%$ del PIB es atribuido a este tipo de negocios (Ga1lo, 1995); un resumen del porcentaje de empresas familiares en distintos países se encuentra en el cuadro No. 1.

\section{\% relativo de Empresas Familiares entre} todos los negocios, por país

$$
\text { País Porcentaje relativo (\%) }
$$

\begin{tabular}{l|l}
\hline Austria & 83.2 \\
\hline Alemania del Este & 82.0 \\
\hline Chile y Australia & $75 \%$ \\
\hline Bélgica & 69.0 \\
\hline Reino Unido & 67.3 \\
\hline Suiza & 67.2 \\
\hline Todo el mundo & 66.0 \\
\hline Finlandia & 63.2 \\
\hline Francia & 60.5 \\
\hline Holanda & 51.7 \\
\hline
\end{tabular}

Fuente: Donckels \& Frohlich, 1991: 154

Cuadro No. 1
En la región centroamericana se sabe que existen unas 3,5 millones de empresas, pero muy poco sabemos sobre su propiedad (Arroyo \& Nebelung: 2002: 49); en el caso particular de Nicaragua, en un estudio recién concluido por una comisión inter-institucional (público-privada) donde participaron la Vice Presidencia de la República, la UNI, el CONICYT, el INPYME, el MIFIC, y CADIN y financiado con recursos de ASDI, se encontró que el $76 \%$ (aproximadamente) de las pymes que estaban innovando en el país eran de carácter familiar; en total el estudio abarcó a 1,005 empresas y con él se creó un software interactivo que muy pronto estará disponible en la página Web del Proyecto de la UNI / BID de empresas familiares.

De acuerdo al dato anterior, si tomamos como base las 550,000 empresas que se calcula existen en el país, podríamos concluir que en Nicaragua hay un parque empresarial de 418,000 negocios familiares.

\section{Tipos de empresas familiares}

Los resultados de numerosos estudios nos enseñan que hay diferentes tipos de empresas familiares, dependiendo del rol de su fundador o de la influencia de la familia empresaria, en resumen se pueden mencionar 3 categorías: control total, ausentismo y de herencia cultural; en la primera, la familia posee las acciones y controla la gerencia de la firma, en la segunda la familia gobierna la empresa pero "subcontrata" profesionales externos para que la manejen, y en la tercera la familia ya perdió el capital pero todavía está involucrada en la gerencia (Lank, 1998).

La empresa familiar también puede ser visualizada desde una perspectiva de etapas por generaciones, y clasificada en 3 fases:

La empresa del héroe, caracterizada por la primera generación con la presencia del fundador, la empresa de los hijos/as, que es representada por los hermanos y hermanas manejando la gerencia, y la empresa de los primos, caracterizada por la presencia de los miembros de las 3ra y 4 ta genera- 
ción, donde familiares y no familiares comparten el mismo lugar de trabajo (Lansberg, 1992).

\section{El rol del fundador}

El fundador juega un rol clave en la creación, desarrollo y preparación del negocio para que entre la siguiente generación; con él/ella se establecen los valores y la cultura que acompañaran al negocio mientras sea de carácter familiar, es por tanto la persona de mayor impacto en la vida de la empresa familiar.

Con mucha frecuencia, el fuerte liderazgo del fundador podría afectar el crecimiento del negocio a mediano y largo plazo por su resistencia a separarse del negocio (Upton, \& Heck, 1997: 248). Algunos autores hablan de 4 estilos distintos de fundadores por la forma como se terminan separando del negocio: el monarca, el general, el embajador y el gobernador.

El monarca, está normalmente a la cabeza de pequeñas empresas, retiene un control cerrado sobre las decisiones estratégicas, y son forzados a salir luego de una larga experiencia. Los generales son también forzados a salir, pero luego "planifican" su retorno. Los embajadores lideran grandes firmas, y reconocen fácilmente su tiempo de salida, y lo hacen con gusto, mantienen contacto con la empresa en una capacidad de consejero. Los gobernadores están a la cabeza en grandes compañías por un tiempo específico y limitado.

Estudios empíricos señalan que la mayoría de los fundadores de empresas familiares caen en las categorías de monarcas o generales, y que cuando se comparan ejecutivos de empresas familiares con no-familiares, los primeros nunca encuentran una razón para salir del negocio, en cambio los segundos están deseosos de retirarse (Upton \& Heck, 1997: 249).

\section{El rol del sucesor}

El sucesor ha sido poco estudiado, pero lo que se sabe es que a la mayoría de los sucesores no les va tan bien cuando heredan el manejo del negocio; un estudio señaló que el 53\% de las empresas más antiguas estaban en un estado de "decline" y la mayoría de ellas manejadas por sucesores y que sólo el $20 \%$ de ellas estaban saliendo adelante.

La mayoría de los sucesores son varones, y la relación padre-hijo mejora cuando el padre entra en los 50's (50-59) y el hijo en los 20's (23-32) y dramáticamente empeora cuando el padre entra en los 60's (60-69) y el hijo en los 30's (34-40) (Upton \& Heck, 1997: 249-250).

\section{La empresa familiar como campo de estudio}

Aún cuando hemos avanzado mucho en el conocimiento de la empresa familiar, todavía queda muchísimo por conocer, especialmente en nuestro contexto, regional y latinoamericano. En Nicaragua sólo se ha hecho un estudio sobre la internacionalización de la empresa familiar, y se espera que el proyecto del BID promueva un marcado interés en el tema de parte de la academia.

\section{El modelo de los 3-círculos}

Una contribución muy importante al estudio de las Empresas familiares fue la de Tagiuri \& David (1982: 42) al construir un modelo basado en la "teoría de sistemas" (Ludwig, 1968) donde hay 3 unidades básicas independientes que operan en la EF y que al hacerlo "invaden" sus respectivos campos. Estos campos son: el de la propiedad, la familia y la firma. Esta última es fuertemente influenciada por las 2 primeras (Figura 1).

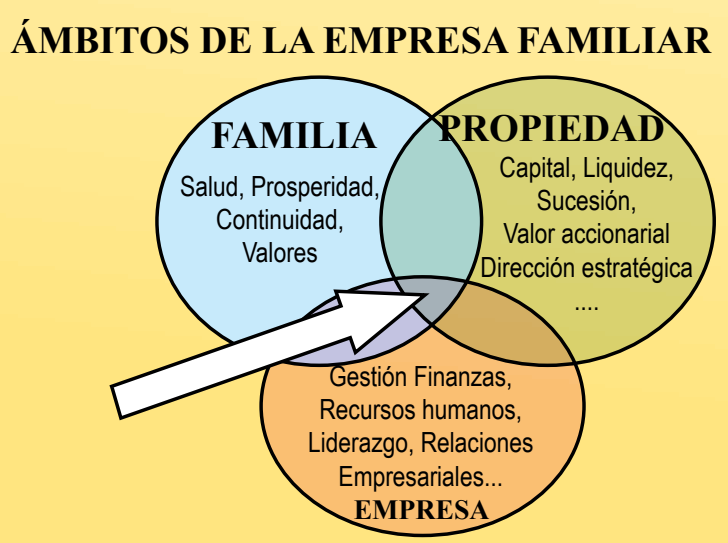

La búsqueda de equilibrio

Figura 1 


\section{El enfoque del Ciclo de Vida}

Otra contribución importante en el estudio de la EF la hicieron Gallo \& Ribeiro (1996) quienes basaron su representación en varios de los conceptos explicados antes. Su modelo sigue una perspectiva generacional de hasta la 3ra generación, que es cuando desaparece la mayoría de las empresas de este tipo (Figura 2).

\section{Etapas en el desarrollo de la EF} Fuente: Gallo \& Ribeiro, 1996

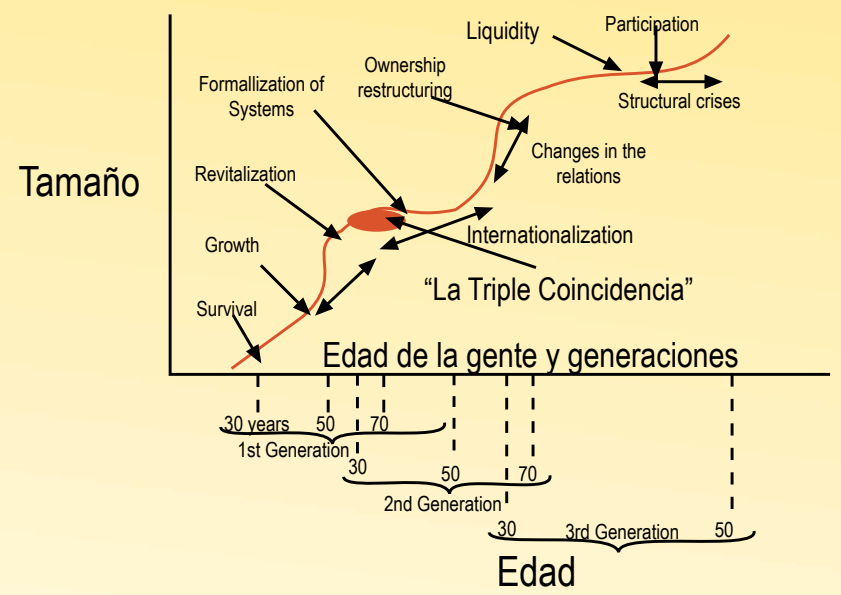

Figura 2

\section{PROYECTO UNI - BID}

Conscientes que la principal problemática de la EF es su sobrevivencia con relación al tiempo, la UNI, con el apoyo del BID, propone apoyar este grupo de empresas por medio de la promoción del fortalecimiento y la competitividad de la PYME nicaragüense (familiar) por medio de la implantación de un modelo de gobierno corporativo que profesionalice su estructura y capacidad de gestión.

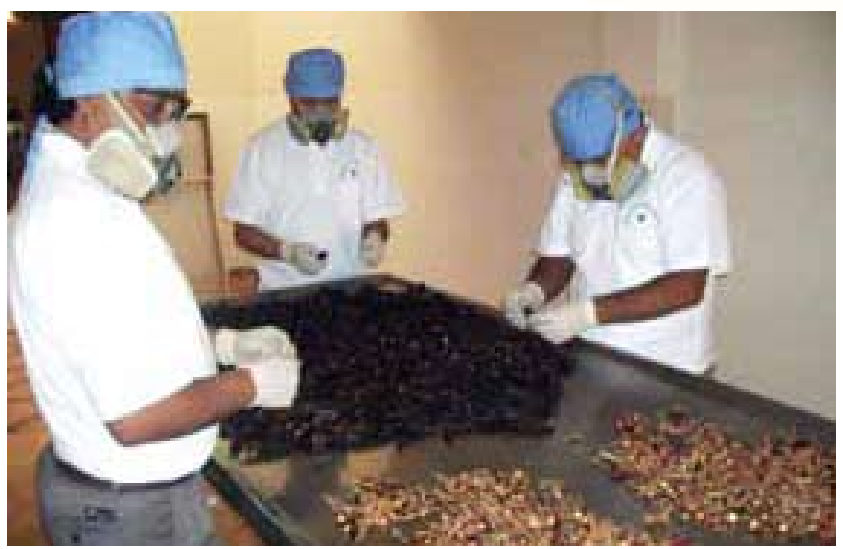

Este proyecto se ha denominado "Promoción e implantación de herramientas de gobierno corporativo en Pymes Nicaragüense" (Proyecto ATN/ ME-11367-NI) y cuenta con recursos por US $\$ 1$ millón, de los que la universidad aporta el 30\% y para lograr el objetivo anterior se ha propuesto desarrollar 4 componentes:

a) Sensibilidad entre profesores, consultores, empresarios, periodistas, y público en general.

b) Capacitación de profesores y consultores, los primeros para que empiecen a impartir la materia de gerencia de empresas familiares en las universidades que muestren interés, y los segundos para que estén preparados para darles asistencia técnica a las empresas de este tipo y puedan implantar en ellas un "kit de herramientas" relacionadas al gobierno corporativo de ellas.

c) Asistencia técnica de los consultores a las Pymes familiares.

d) Sistematizar la experiencia.

\section{CONCLUSIONES}

Las empresas familiares son las unidades empresariales más numerosas en cualquier contexto, y juegan un rol clave en el empleo, primero de la familia empresaria, y segundo asegurando el empleo de más de la mitad de los habitantes de cualquier país; lo mismo puede decirse de su contribución al PIB, generación de riqueza, creación de nuevos negocios y las exportaciones.

Sin embargo, su modelo actual de operación no les está permitiendo pasar más allá de la 3ra y 4ta generación, poniendo en peligro su sobrevivencia, y con ello su impacto positivo a la sociedad.

Este reto es ocasionado, principalmente por causas internas, con que cuenta el negocio, entre las que están el estilo de planificación, aquellos aspectos relacionados con la sucesión, el rol y el 
estilo del fundador, del sucesor, la formación de los sucesores, confusión entre los roles de propietario y gerente, confusión entre el interés de la familia y de la empresa, atraso en el proceso de sucesión, crecimiento e internacionalización de la empresa, desarrollo de sucesores, conflictos entre miembros familiares, la planificación estratégica del negocio, temas de riqueza y propiedad, la profesionalización, gobierno corporativo, y enfoque de género, entre otros.

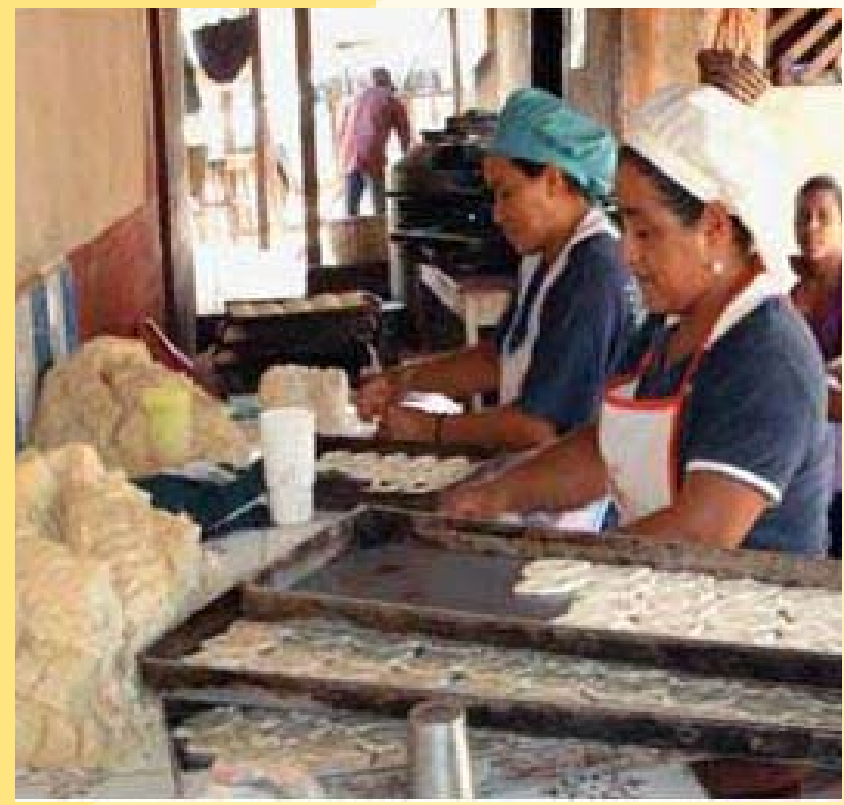

Aun cuando se han puesto las bases para el estudio de este tipo de empresas, hace falta mucho por conocer, principalmente desde la perspectiva de los países en vías de desarrollo, en cuyas latitudes se encuentra la región centroamericana; para enfrentar nuestros retos particulares, la UNI ha iniciado un proyecto, financiado con fondos propios y del BID para atender este tipo de negocios, por lo que este proyecto espera en sus 3 años despertar el interés por el tema, sensibilizar a los distintos grupos metas (enfoque ecológico), capacitar a profesores y consultores, y darle asistencia técnica luego a las empresas de familia.

Si tomamos como base las más de 418,000 empresas familiares que existen en Nicaragua, esto nos da una idea de la dimensión del trabajo que tenemos por delante.

\section{BIBLIOGRAFÍA}

1. Arroyo, J. \& Nebelung, M. (2002): "La micro y pequeña empresa en América Centra: realidad, mitos y retos", GTZ/PPROMOCAP \& PROMICRO/ OIT, Guatema.a

2. Donelly, Robert G. (1964): "The family business", Harvard Business Review, 1, pp. 427-445

3. Donckels, R. Frohlich, E (1991): “Are family businesses really different? European experiences from STRATOS”, Family Business Review, 4, pp. 149-160

4. Gallo, M A (1995): "The role of family business and its distinctive characteristics behavior in industrial activity", Family Business Review, Vol. 2, No. 2, Summer

5. Gallo, M.A. \& García Pont (1989): "La empresa familiar en la economía española", Papeles de Economía Española, 39, pp. 67-85

6. Ludwig, Von Bertalanffy (1968): “General System Theory: Foundations, Development, Applications", NY, Braziller

7. Tagiuri, R. \& Davis, J.A. (1992): “On goals of successful family companies". Family Business Review, 5, pp 43-62.

8. Upton, N. B. \& Hech, R. K. Z. (1997): “The family business dimension of entrepreneurship", en Sexton, D. L. \& Smilor, R. W. (editors) (1997): "Entrepreneurship 2000", Upstart Publishing Co., p. $243-266$

9. Ward, J. L. (1987): "Keeping the family business healthy", San Francisco, CA: Jossey-Bass

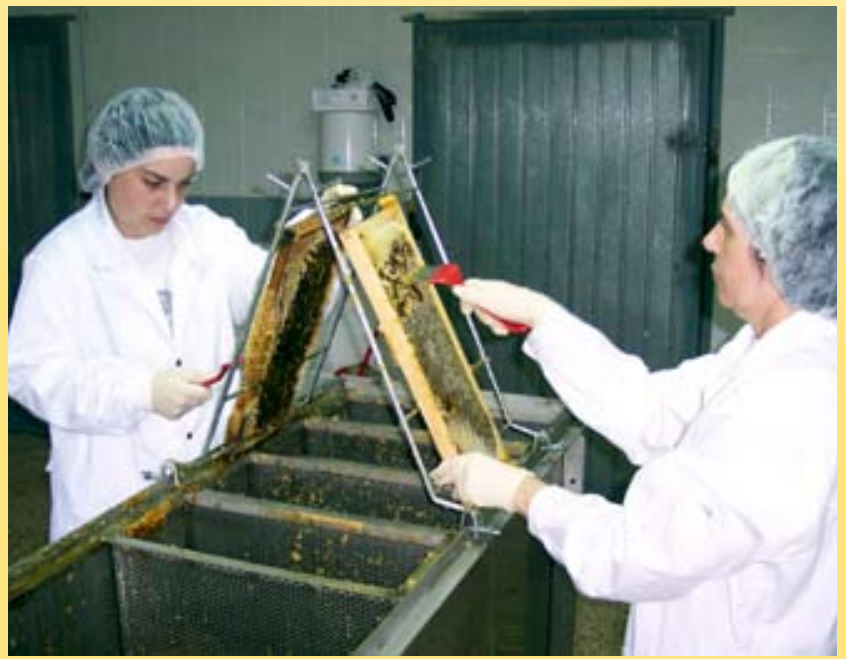

